\title{
Formation and decay of toroidal and bubble nuclei and the nuclear equation of state
}

\author{
H. M. Xu, J. B. Natowitz, C. A. Gagliardi, and R. E. Tribble \\ Cyclotron Institute, Texas A\&M University, College Station, Texas 77843 \\ C. Y. Wong \\ Physics Division, Oak Ridge National Laboratory, Oak Ridge, Tennessee 37831 \\ W. G. Lynch \\ National Superconducting Cyclotron Laboratory, Michigan State University, East Lansing, Michigan 48824
}

(Received 9 November 1992)

\begin{abstract}
Multifragmentation, following the formation of toroidal and bubble nuclei, is observed with an improved Boltzmann-Uehling-Uhlenbeck model for central ${ }^{92} \mathrm{Mo}+{ }^{92} \mathrm{Mo}$ collisions. With a stiff equation of state, simultaneous explosion into several nearly equal fragments in a ringlike manner occurs due to the formation of metastable toroidal nuclei. In contrast, with a soft equation of state, simultaneous explosion into several nearly equal fragments in a volumelike manner occurs due to the formation of metastable bubble nuclei. Experimental signatures for the formation of these exotic shapes are discussed.

PACS number(s): 25.70.Pq, 21.65.+f, 24.10.Cn, 25.70.Gh
\end{abstract}

Two decades ago, in connection with the stability of nuclei with new types of topology proposed by Wheeler [1] and Siemens and Bethe [2], Wong systematically studied the instabilities of toroidal and spherical bubble nuclei within the context of liquid drop, shell, and Hartree-Fock models [3]. He subsequently argued that higher nuclear temperatures enhance the possibility for the formation of toroidal and bubble nuclei [4]. Toroids have been seen in collisions between water drops [5]. Bubbles were observed in one-dimensional hydrodynamical collisions $[6,7]$ as well as in hot dense matter $[8,9]$ and Thomas-Fermi and Hartree-Fock calculations [10]. Recently, theoretical research in this area was revived when it was suggested that the formation of toroids and bubbles could provide new decay modes for multifragment disintegration [11-15].

To investigate the dependence on the equation of state (EOS) and to look for experimental observables, we have performed improved Boltzmann-Uehling-Uhlenbeck (BUU) calculations [15-17] for ${ }^{92} \mathrm{Mo}+{ }^{92} \mathrm{Mo}$ collisions. In our calculations, we have included Coulomb interactions and have used a lattice Hamiltonian method [18] to propagate test particles. In the present calculations for the ${ }^{92} \mathrm{Mo}+{ }^{92} \mathrm{Mo}$ system, we find that, depending on the nuclear equation of state, a metastable toroidal or bubble nucleus can be formed. The decays of these unstable states are simultaneous, yet very slow. We predict a significant increase in the cross sections of nearly equal fragments with small center-of-mass energies as a result of the cold breakup of a bubble or toroidal nucleus. These two shapes can further be separated by the coplanarity of the nearly equal fragments with similar energies, which, in turn, could provide information about the nuclear equation of state.

We simulate the Boltzmann-Uehling-Uhlenbeck equation [19]

$\frac{\partial f_{1}}{\partial t}+\mathbf{v} \cdot \nabla_{r} f_{1}-\nabla_{r} U \cdot \nabla_{p} f_{1}=\frac{4}{(2 \pi)^{3}} \int d^{3} k_{2} d \Omega \frac{d \sigma_{n n}}{d \Omega} v_{12}\left[f_{3} f_{4}\left(1-f_{1}\right)\left(1-f_{2}\right)-f_{1} f_{2}\left(1-f_{3}\right)\left(1-f_{4}\right)\right]$

with the lattice Hamiltonian method of Lenk and Pandharipande [18]. In Eq. (1), $\frac{d \sigma_{n n}}{d \Omega}$ and $v_{12}$ are the inmedium cross section and relative velocity for the colliding nucleons, and $U$ is the total mean-field potential consisting of the Coulomb potential and a nuclear potential with isoscalar and symmetry terms [16]. In our calculations, we use two parameter sets [19] for the EOS which correspond to values of nuclear compressibility at $K=200 \mathrm{MeV}$ (soft EOS) and $K=375 \mathrm{MeV}$ (stiff EOS), respectively. For simplicity, $\sigma_{N N}=\int \frac{d \sigma_{n n}}{d \Omega} d \Omega$ is chosen to be isotropic and energy independent [19]. The meanfield and the Pauli-blocking factors in the collision integral are averaged over an ensemble of 80 parallel simulations.
In Figs. 1 and 2, we display the results of BUU calculations for ${ }^{92} \mathrm{Mo}+{ }^{92} \mathrm{Mo}$ collisions at $b=0$ and $E / A=85$ $\mathrm{MeV}$ (Fig. 1) and $100 \mathrm{MeV}$ (Fig. 2) calculated with the stiff EOS. For both calculations, a compressed state is observed at $t \approx 30 \mathrm{fm} / c$. This state subsequently expands in the direction perpendicular to the beam and forms a dilute system of oblate shape at $t=60 \mathrm{fm} / c$. From this moment on, the system contracts locally and a hole starts to emerge from the center at $t \approx 90 \mathrm{fm} / c$ and this hole continues to expand outward very slowly, while keeping the overall size unchanged. Thus a metastable torus is gradually formed with its normal axis parallel to the beam direction. This torus eventually breaks up simultaneously, though slowly, into fragments of nearly 


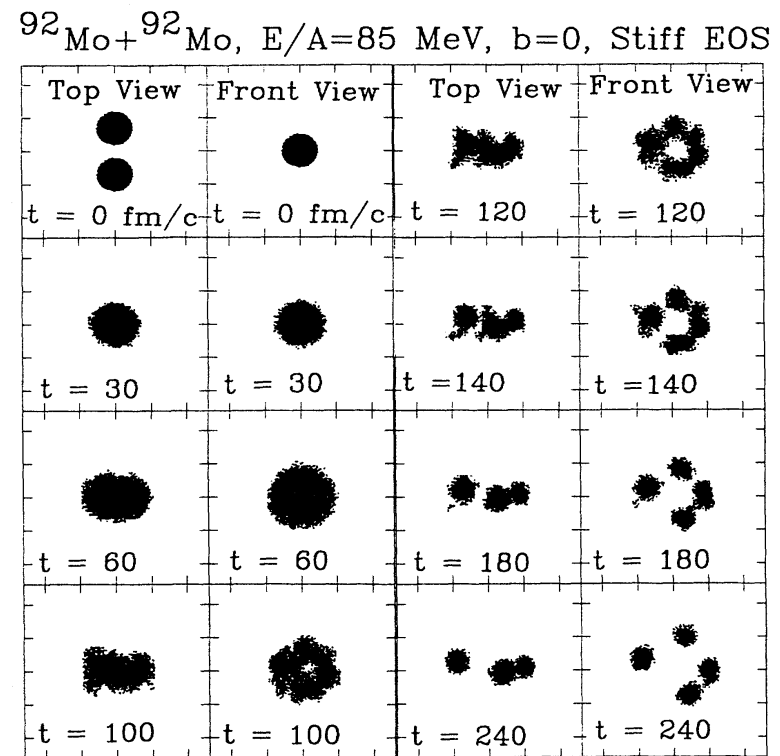

FIG. 1. BUU calculations with the stiff EOS for ${ }^{92} \mathrm{Mo}+{ }^{92} \mathrm{Mo}$ collisions at $E / A=85 \mathrm{MeV}, b=0$. Only areas with densities $\rho \geq 0.1 \rho_{0}$ are shown. The scales between neighboring ticks are $10 \mathrm{fm}$.

equal sizes with their radii approximately equal to the minor radius of the torus at breakup (for later reference, we characterize the torus by an aspect ratio $R / d[3]$ where the major radius $R$ is measured from the center of torus to the center of the circular meridian and the minor radius $d$ is the radius of the meridian). Since these coplanar fragments separate with velocities approximately in the radial directions, they will be accelerated by their mutual Coulomb forces and maintain their coplanarity until they reach the detectors. As the incident energy is increased, a torus with a larger aspect ratio is formed which subsequently breaks up into a larger number of fragments (see Fig. 2). We will come back to this point.

In contrast, in the calculations with the soft EOS (see

$92{ }_{\mathrm{Mo}}+{ }^{92} \mathrm{Mo}, \mathrm{E} / \mathrm{A}=100 \mathrm{MeV}, \mathrm{b}=0$, Stiff EOS

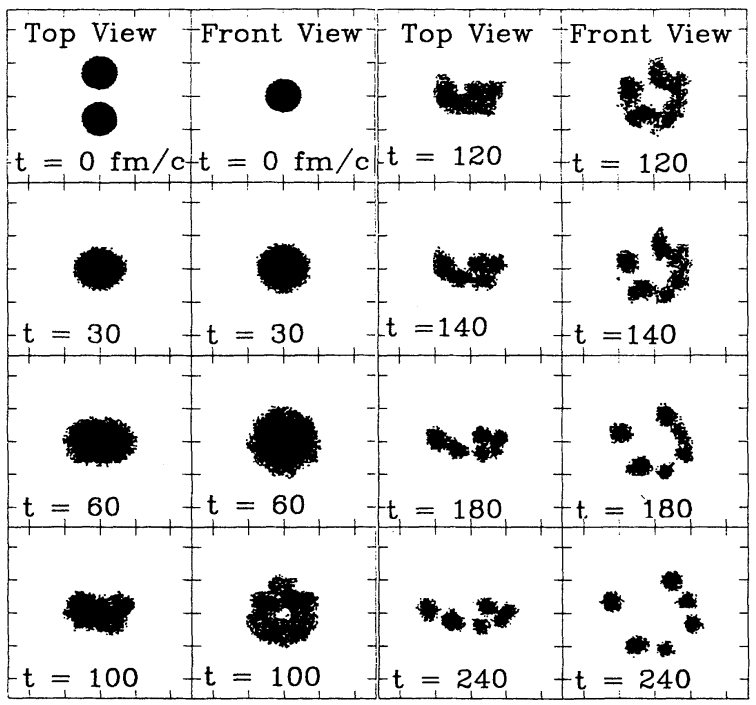

FIG. 2. Same as Fig. 1 , but calculated at $E / A=100 \mathrm{MeV}$.
Fig. 3), a nuclear bubble starts to emerge when the system expands to its maximum at $t \approx 60 \mathrm{fm} / c$. Similar to the formation of a torus, the inner surface of the bubble starts from zero and continues to increase, while the outer surface remains relatively unchanged. This bubble stays for $t \approx 60 \mathrm{fm} / c$ and then breaks up simultaneously into several fragments (fragments are emitted isotropically).

The theoretical interpretations concerning the formation and decay of these exotic shapes have been discussed earlier, in a one-dimensional model, by Bertsch and Mundinger [7], and, recently, in Refs. [11-14]. Here we focus our study on the dependence on the EOS and on looking for experimental observables. Clearly, as one can see from Figs. 1 and 2, an explosion due to the formation of a metastable torus (calculated by the stiff EOS) would be characterized by nearly coplanar emission of nearly equal fragments. On the other hand, an explosion due to the formation of a metastable bubble nucleus (soft EOS) would be characterized by isotropic emission of nearly equal fragments. In both cases, however, the kinetic energies of the emitted fragments in the center of mass appear very small compared to the incident energy. This reflects the fact that most of mass and excitation energy is carried away by emission of particles before the toroidal and bubble nuclei break up [20]. Thus these nearly equal fragments resulting from the decay of a torus or a bubble will be focused to small angles in the laboratory frame.

Because of the lack of higher-order many-body correlations and fluctuations in the BUU model (mean-field + two-body collisions), the fragments formed after the onset of multifragmentation $\left(t_{\text {breakup }} \approx 120 \mathrm{fm} / c\right)$ may not be viewed literally as realistic fragments. To guide experimental efforts for searching for the formation of toroidal nuclei, we estimate the kinematics of the final fragments prior to the decay of the toroidal nuclei as follows. Assuming a toroidal nucleus breaks up into $\lambda$ identical fragments, we then estimate the kinetic energy per nucleon for each fragment by evaluating

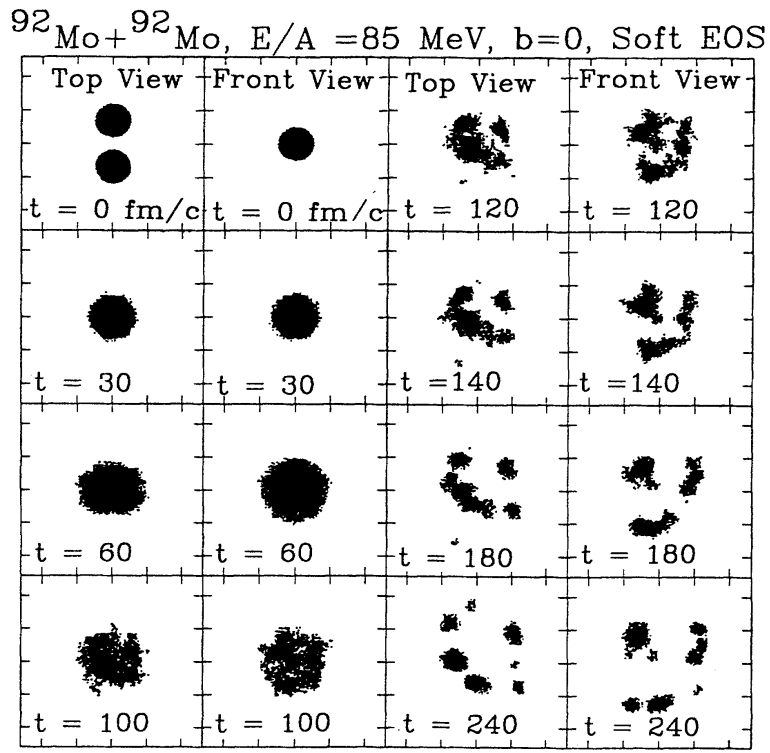

FIG. 3. Same as Fig. 1, but calculated with the soft EOS. 


$$
E_{K}(r=\infty) / A \approx\left[E_{K}\left(r=R_{\text {breakup }}\right)+V_{\text {Coul }}\left(r=R_{\text {breakup }}\right)-\lambda^{-2 / 3} \frac{3}{5} \frac{Z_{t}^{2}}{r_{0} A_{t}^{1 / 3}}\right] / A_{t}
$$

where $Z_{t}$ and $A_{t}$ indicate, respectively, the charge and mass of the toroidal nucleus at breakup, calculated at $t=120 \mathrm{fm} / c$. The first and the second terms, estimated numerically from the BUU calculation, depict the radial kinetic energy and Coulomb energy, respectively, at breakup. The third term is the Coulomb self-energy for $\lambda$ fragments. We further estimate the various components of excitation energies using techniques outlined in Ref. [21] and find that the toroidal nuclei formed are quite cold, with thermal excitation energies per nucleon, $E_{\text {thermal }} / A \approx 1-2 \mathrm{MeV}[15]$, at breakup $(t \approx 120 \mathrm{fm} / c)$. Thus the breakup process is a cold breakup process at low temperatures similar to the cold scission of an initially hot system well understood in fission processes. Because of the geometry of the toroids and because of cold breakup at low temperatures, the decay fragments will have approximately similar masses, thus enhancing the cross sections for fragments with nearly equal sizes at kinematic regions discussed below.

Guided by the BUU calculations, we predict a typical case of fragmentation into three ${ }^{20} \mathrm{Ne}$ in a coplanar final state for ${ }^{92} \mathrm{Mo}+{ }^{92} \mathrm{Mo}$ collisions at $E / A=75$ $\mathrm{MeV}$, each with kinetic energy per nucleon in the c.m. frame, $E_{k} / A \approx 1.8 \mathrm{MeV}$ or a total energy of $36 \mathrm{MeV}$ per ${ }^{20} \mathrm{Ne}$ fragment. These energies are very small, and the fragments will therefore be focused to laboratory angles $\theta_{\text {lab }} \lesssim \theta_{\max } \approx 16.7^{\circ}$. At higher energies, we predict typical cases of four ${ }^{12} \mathrm{C}$ and five ${ }^{7} \mathrm{Li}$ fragments, respectively, at $E / A=85$ and $100 \mathrm{MeV}$. On the average, as the incident energy is increased, a larger number of fragments with smaller mass per fragment are emitted to larger critical laboratory angles $\theta_{\max }$. This is because the higher the incident energy, the larger the collective expansion, and, therefore, the larger the aspect ratio of the torus which is formed. This larger torus will subsequently decay into more fragments with smaller masses. We emphasize here that the specific values of the multiplicities listed in Table I are used for the convenience of estimating the kinematics. In reality, large fluctuations in fragment multiplicities are expected, which is beyond what the BUU model can predict. However, although the kinetic energy may depend on the mass and multiplicity, each fragment should have approximately the same c.m. kinetic energy per nucleon; thus, these nearly equal fragments are focused to angles less than $\theta_{\text {lab }} \lesssim 20^{\circ}$. This result is very important, because there are already multifragmentation data published in several systems [22-26]. To investigate whether the toroidal or bubble geometry is formed, a reanalysis of these data to search for the enhancement of fragments with nearly equal masses in the kinematical regions as suggested in Table $I$ is called for.

Additional dependence on the impact parameter could complicate experimental studies. To investigate this dependence, we have performed extensive calculations to investigate the dependence of emission pattern on the impact parameter. We find that, instead of an emission plane perpendicular to the beam direction, the emission plane (oblate shape when calculated with the stiff EOS) is rotated by an angle whose value depends on the impact parameter at impact parameters $b<3 \mathrm{fm}$ [15]. For the calculation with the soft EOS, an emission pattern corresponding to a prolate shape is found. For both cases, the kinetic energies in the center of mass for the final fragments are surprisingly small and they are emitted at angles $\theta_{\text {lab }} \lesssim 20^{\circ}$ with kinematics similar to that listed in Table I. Because of the toroidal and bubble geometries and because of low temperatures at freeze-out, the fragments emitted in central collisions $(b<3 \mathrm{fm})$ will be nearly equal in size. Thus, in addition to the enhancement for fragments with nearly equal sizes at forward angles, an analysis of the event shapes of these nearly equal fragments, event by event, could provide information concerning the geometry of the sources (toroidal or bubble). This, in turn, could provide information about the equation of state. At large impact parameters, $b \gtrsim 3 \mathrm{fm}$, whether the toroidal or bubble geometry is formed becomes questionable and one observes remnants of the projectilelike and targetlike residues. Clearly, these peripheral collisions, where projectilelike and targetlike heavy residues are emitted, differ significantly from the central collisions, where the bubble or toroidal geometry is formed and one observes several fragments of nearly equal sizes with very small c.m. energies.

In summary, with our improved BUU model, we predict multifragmentation following the formation of metastable toroidal and bubble nuclei in ${ }^{92} \mathrm{Mo}+{ }^{92} \mathrm{Mo}$ collisions. We find that the multifragmentation pattern depends sensitively on the nuclear equation of state. With a stiff equation of state, simultaneous explosion into several nearly equal fragments in a ringlike manner occurs due to the formation of metastable toroidal nuclei. In contrast, with a soft equation of state, simultaneous explosion into several nearly equal fragments in a volumelike manner occurs due to the formation of metastable bubble nuclei. Based on our numerical simulations, we propose the following signatures for detecting the new phenomena: (1) Because of the geometries of bubbles and toroids and because of the cold breakup at low temperatures, we predict enhanced cross sections for fragments with nearly equal masses at small center-of-mass ener-

TABLE I. Multifragment decay from a metastable toroidal nucleus in ${ }^{92} \mathrm{Mo}+{ }^{92} \mathrm{Mo}$ collisions predicted by our improved BUU model

\begin{tabular}{cccccc}
\hline \hline $\begin{array}{c}E / A \\
(\mathrm{MeV})\end{array}$ & $\begin{array}{c}\text { Fragment } \\
(\mathrm{MeV})\end{array}$ & $\begin{array}{c}\text { Multiplicity } \\
(\mathrm{MeV})\end{array}$ & $E_{k} / A$ & $E_{k}$ & $\theta_{\max }$ \\
\hline 75 & ${ }^{20} \mathrm{Ne}$ & 3 & 1.8 & 36 & $16.7^{\circ}$ \\
85 & ${ }^{12} \mathrm{C}$ & 4 & 2.4 & 29 & $19.1^{\circ}$ \\
100 & ${ }^{7} \mathrm{Li}$ & 5 & 2.9 & 20 & $20.9^{\circ}$ \\
\hline \hline
\end{tabular}


gies. Because of the small c.m. kinetic energies, these nearly equal fragments are therefore focused to angles $\theta_{\text {lab }} \lesssim 20^{\circ}$ (Table I). (2) The coplanarity of the emission pattern for the intermediate mass fragments with nearly equal masses and energies at forward kinematics $\left(\theta_{\text {lab }} \lesssim 20^{\circ}\right)$ may carry important information concerning the geometry of the sources. This, in turn, could provide information about the stiffness of the equation of state. Obviously, higher-order many-body correlations and fluctuations, not incorporated in our BUU model, as well as thermal and Fermi motions, could influence our detailed predictions (listed in Table I). Further investigations are also required to assess the sensitivity of the detailed algorithm of Pauli blocking, dependence on the number of test particles, momentum-dependent interactions, and surface energy to experimental observables. Nonetheless, our predictions should provide a guidance for experimental efforts to search for these new mechanisms. Moreover, experimental testing of these predictions can provide a very stringent test of one-body BUU models and could lead to the evidence for the existence of toroidal or bub- ble nuclei, and thus information concerning the stiffness of the equation of state.

Note added. After we submitted the paper, we became aware of a paper by Bruno et al. [27] where they reported a significant enhancement for fragments with nearly equal masses at kinematical regions similar to our predictions. Though these authors have not associated their data with exotic shapes, their data, nonetheless, could be the first experimental evidence for the formation of bubble or toroidal geometry in heavy-ion collisions.

We would like to acknowledge P. Danielewicz, C.K. Gelbke, C.M. Ko, L.G. Moretto, R. Schmitt, D.E. Youngblood, and Y.M. Zheng for helpful discussions. This work was supported in part by the U.S. DOE under Grant No. DE-FG05-86ER40256 and Contract No. DE-AC05-84OR21400 managed by Martin Marietta Energy Systems, Inc, the Robert A. Welch Foundation, and the NSF under Grant No. PHY-89-13815. W.G.L. acknowledges the financial support of the U.S. Presidential Young Investigator Program.
[1] J.A. Wheeler, Nucleonics Notebook, 1950 (unpublished).

[2] P. Siemens and H. Bethe, Phys. Rev. Lett. 18, 704 (1967).

[3] C.Y. Wong, Phys. Lett. 41B, 446 (1972); 41B, 451 (1972); K.T.R. Davies et al., ibid 41B 455 (1972); C.Y. Wong, Ann. Phys. (N.Y.) 77, 279 (1973).

[4] C.Y. Wong, Phys. Rev. Lett. 55, 1973 (1985).

[5] J.R. Adams et al., J. Appl. Phys. 39, 5173 (1968).

[6] C.Y. Wong et al., Phys. Lett. 66B, 19 (1977).

[7] G. Bertsch and D. Mundinger, Phys. Rev. C 17, 1646 (1978).

[8] J. Lattimer et al., Nucl. Phys. A432, 646 (1985).

[9] H. Reinhardt et al., Nucl. Phys. A432, 630 (1985).

[10] J. Nemeth et al., Z. Phys. A 320, 691 (1985).

[11] L.G. Moretto et al., Phys. Rev. Lett. 69, 1884 (1992).

[12] W. Bauer et al., Phys. Rev. Lett. 69, 1888 (1992).

[13] D.H.E. Gross et al., Ann. Phys. (Leipzig) 1, 467 (1992).

[14] B. Borderie et al., Phys. Lett. B 302, 15 (1993).

[15] H.M. Xu, Phys. Rev. C 46, R2144 (1992); H.M. Xu et al. (unpublished).

[16] H.M. Xu et al., Phys. Rev. Lett. 65, 843 (1990); Phys. Lett. B 261, 240 (1991); 299, 199 (1993).
[17] H.M. Xu, Phys. Rev. Lett. 67, 2769 (1991); Phys. Rev. C 46, R389 (1992); 47, 891 (1993).

[18] R.J. Lenk and V.R. Pandharipande, Phys. Rev. C 39 , 2242 (1989).

[19] G.F. Bertsch and S. Das Gupta, Phys. Rep. 160, 189 (1988), and references therein.

[20] The residue, either the torus or the bubble, which survives the initial collision, decays in a much slower time scale. Therefore, the decay products (the nearly equal fragments) have much smaller transverse energies than those observed recently [22-26], because they are focused to small angles in the beam direction, and may not be separated, in the data, from those which are emitted before the formation of the residues.

[21] B. Remaud et al., Nucl. Phys. A488, 423c (1988).

[22] C.A. Ogilvie et al., Phys. Rev. Lett. 67, 1214 (1991).

[23] R.T. de Souza et al., Phys. Lett. B 268, 6 (1991).

[24] D.R. Bowman et al., Phys. Rev. Lett. 67, 1527 (1991).

[25] Y. Blumenfeld et al., Phys. Rev. Lett. 66, 576 (1991).

[26] K. Hagel et al., Phys. Rev. Lett. 68, 2141 (1992).

[27] M. Bruno et al., Phys. Lett. B 292, 251 (1992). 